\title{
Preoperative imaging for breast conservation surgery-do we need more than conventional imaging for local disease assessment?
}

\author{
Eugene Ong \\ Diagnostic Radiology, Mount Elizabeth Novena Hospital, Singapore \\ Correspondence to: Eugene Ong. Diagnostic Radiology, Mount Elizabeth Novena Hospital, Singapore. Email: eugenemwong@hotmail.com.
}

\begin{abstract}
Breast conservation surgery (BCS) is offered for early breast cancer. Conventional imaging with mammography and ultrasound would have been performed prior to surgery. This article considers other imaging modalities available [such as 3D tomosynthesis, magnetic resonance imaging (MRI), contrastenhanced spectral mammography, positron emission mammography (PEM), breast-specific gamma imaging (BSGI) and cone beam computed tomography (CBCT)] and discusses the evidence for these in terms of diagnostic accuracy and clinical outcomes.
\end{abstract}

Keywords: Preoperative imaging; breast conservation surgery (BCS)

Submitted Mar 21, 2018. Accepted for publication Aug 20, 2018.

doi: 10.21037 /gs.2018.08.05

View this article at: http://dx.doi.org/10.21037/gs.2018.08.05

\section{Background}

Breast conservation surgery (BCS) has become the standard of care for early stage breast cancer. It has been shown to have equivalent survival rates to mastectomy (1-3).

This involves complete excision of the tumor with histologically negative margins to reduce the risk of local recurrence. Close or positive margins would result in further surgery which may involve margin re-excision or mastectomy. This would be undesirable for the patient and may result in a poorer cosmetic outcome while incurring higher costs.

Important considerations to achieve successful BCS would then include determination of lesion size, disease extent (including multifocal and multicentric disease), involvement of adjacent structures to the lesion such as overlying skin, nipple and underlying chest wall structures. Staging of the cancer to include ipsilateral and contralateral axillary lymphadenopathy as well as lymphadenopathy in other areas such as the internal mammary and supraclavicular regions as well as distant disease would also be performed.

At the time of surgery, conventional imaging with mammography, breast ultrasound or both would usually have been performed. These may be part of the initial screening process which detected the tumor, part of the initial investigation for symptomatic patients with breast lumps or nipple discharge and may also have provided imaging guidance for the biopsy procedure leading to histological confirmation.

To complete staging of the disease, further imaging is then performed prior to surgery. These may include chest radiographs, abdominal ultrasound, computed tomography (CT) and PET-CT studies.

To assess local disease extent, other imaging modalities may be employed. These include (but are not limited to) 3D digital breast tomosynthesis (DBT), breast MRI, positron emission mammography (PEM), breast-specific gamma imaging (BSGI), cone beam computed tomography (CBCT) and contrast enhanced spectral mammography (CESM).

In the assessment of local disease, the theoretical background of breast cancer needs to be considered. The sick lobe hypothesis proposed by Tot (4) considers breast carcinoma to be a lobar disease with similar changes simultaneously or asynchronously appearing in situ or invasive tumor foci developing within a single sick lobe. Early stage disease is relatively confined to the lobe before spreading beyond it. This is supported by the appearance on large format histological sections which demonstrate subgross 
distribution of the lesion in various patterns within the lobe.

In line with this hypothesis, lobar ultrasound of the breast with ductal echography suggested by Teboul (5) and Amy (6) results in better visualization of the disease distribution correlating with histopathological sections.

Given this hypothesis, consideration of advance modalities which better depict the disease extent within the sick lobe or even beyond it may be useful in preoperative evaluation.

\section{D DBT}

DBT improves diagnostic accuracy and reader confidence when identifying benign and malignant lesions. It has also been shown to be more accurate than 2D digital mammography in assessing tumour size and in the assessment of multifocal tumours.

In a pre-operative setting, studies show DBT to be superior to mammography and ultrasound in the assessment of breast tumour size (7). It is also superior to mammography in detecting multifocal disease although MRI is more sensitive (8).

In dense breasts, DBT may also significantly improve preoperative staging compared to mammography alone with limitations in cases of invasive lobular carcinoma (ILC) (9).

There is however little if any literature on the effect of DBT on breast conservation surgical outcomes to date.

\section{Magnetic resonance imaging (MRI)}

Breast MRI in the preoperative setting remains a controversial subject.

Theoretically, breast MRI should be superior in evaluating disease extent as it provides multiplanar structural information about the tumor and its relative location within the breast and surrounding structures. It also allows functional assessment of the lesion with diffusion-weighted imaging as well as dynamic contrast enhancement.

The contralateral breast may also be scanned at the same time along with the axillary and internal mammary regions on both sides.

Studies have shown that MRI undoubtedly detects additional disease and accurately detects chest wall invasion.

Meta-analysis of data from nineteen studies showed MRI detects additional disease in $16 \%$ of women with breast cancer. This led to conversion from wide local excision (WLE) to mastectomy in $8.1 \%$, from WLE to more extensive surgery was $11.3 \%$ in multifocal/multicentric disease (10).
Meta-analysis of twenty-two studies reported contralateral malignancies detected only by MRI showed that MRI detects abnormalities not seen on conventional imaging in $9.3 \%$ of women. Excluding false positive MRI-only detected lesions, the incremental cancer detection rate for MRI is $4.1 \%$ (11).

When compared to cancers detected on screening ultrasound, MR detects an additional 5.6 percent of disease (12).

Suspected chest wall involvement was prospectively studied in 19 patients with posteriorly located breast tumors suspected to involve the pectoralis major muscle based on either mammography or clinical exam. MRI results were compared with surgical and pathologic findings. The presence of abnormal enhancement within the pectoralis major muscle on MRI was $100 \%$ sensitive and $100 \%$ specific for identifying tumors that actually involved the pectoralis major muscle (13).

The controversy is whether the increased detection of lesions translates to better clinical outcomes. This would include lower re-operative rates to excise residual disease, lower recurrence rates following surgery, lower contralateral metachronous tumors as well as better patient survival outcomes.

The first two randomized control trials (RCTs) were COMICE (14) and MONET (15). COMICE which was performed in the UK show similar reoperation rates in the groups with and without preoperative MRI. MONET showed increase reoperation rates in the MRI group.

The meta-analysis of 9 eligible studies (2 RCTs, 7 comparative cohorts) showed that MRI significantly increased mastectomy rates with no significant difference in re-excision rates. There was weak evidence that MRI reduced re-excision surgery in patients with ILC (16).

Despite this, there have also been other studies showing lower re-excision rates in women who underwent preoperative breast MRI but no statistically significant effect on regional recurrence or disease-free survival (17).

In a Swedish RCT (18), pre-operative breast MRI altered treatment plan in $18 \%$ and conversion to mastectomy was $20 \%$ in the MRI group (vs. $10 \%$ in the control group). Reoperation rates were lower in the MRI group (5\%) compared to the control group (15\%).

A large retrospective study (19) showed that in ILC, preoperative MRI had lower odds ratio of reoperation after initial BCS.

Critical review of the clinical outcome studies by Causer (20) may help to explain and put into perspective some of the controversies raised.

To explain why higher cancer detection rate does not 
translate to better clinical outcomes, it is argued that the higher cancer detection rate by using breast MRI is much greater than breast recurrence rates after BCS and modern adjuvant therapies, leading to surgical overtreatment of biologically irrelevant cancer foci that would otherwise be treated with adjuvant therapies.

The COMICE clinical trial assessing the use of preoperative MRI had limitations including variable levels of expertise in performing breast MRIs, variability in interpretive skills, and lack of standardization in surgical application of MRI findings. In addition MRI guided biopsy was not widely available which may have led to unnecessary mastectomies (38\% of conversions to mastectomy based on false positive MRI findings).

The outcome of BCS in terms of how much additional tissue was resected in obtaining negative margins was also not assessed and wider excisions than necessary may have been performed than necessary to this end at the expense of cosmesis (21).

To address some of these issues, the American college of radiology has established practice guidelines (22) which require certain standards of performing and interpreting breast MRI and recommendation that MRI guided intervention be available to evaluate lesions only seen on the MRI prior to surgery.

The utility of preoperative breast MRI in the context of neo-adjuvant chemotherapy is a special consideration.

The most important use of MRI would be to reliably identify patients whose tumors are not responding to neoadjuvant chemotherapy to stop or change the therapy regimen to avoid added morbidity associated with continued ineffective chemotherapy.

MRI would also need to avoid false positive findings which would result in premature discontinuation of effective chemotherapy.

Meta-analysis of 44 studies (23) showed that post chemotherapy, MRI has been shown to accurately detect residual tumor and is more accurate than mammography.

In cases where preoperative MRI detects additional lesion not seen on conventional imaging, additional evaluation with second look ultrasound and subsequent biopsy under ultrasound or MRI guidance may cause delays in breast conserving surgery.

Bleicher et al. (24) found a 22.4-day delay in pretreatment evaluation for patients receiving MRI. The delay was 11.1 days in a study by Zhang et al. (25).

However, in a separate study, Vreeland et al. (26) found no delay to initial surgical therapy and, perhaps more importantly, a slight decrease in time to margin-negative surgical therapy in the MRI group.

The group proposed that rather than basing merit of a pre-operative test on how quickly a patient undergoes an initial operation, a better metric might be time to completion of surgical treatment (to include reoperation for positive margins).

The difference was attributed to their unique setting where all patient care from imaging to image-guided biopsies being in one centre with comprehensive insurance coverage of diagnostic tests. This allows a standardized protocol allowing pre-operative MRI to be completed without any delay in care.

While the sensitivity of MRI for breast cancer is unparalleled, its preoperative use in all patients does not appear justified as there is no evidence that patient outcomes are improved with identification of additional ipsilateral or contralateral occult malignancies. Preoperative breast MRI has shown usefulness in patients with ILC and in patients for whom neoadjuvant chemotherapy is planned. Further studies (such as ACRIN 6694) may demonstrate effectiveness of preoperative MRI in select patient populations, such as those with genetic mutations, and for patients with cancers with specific molecular profiles such as triple negative and Her2 positive cancers.

\section{CESM}

CESM is a mammographic technique in which an iodinebased contrast agent is administered intravenously two minutes prior to image acquisition.

The procedure uses a low-energy image, similar to full field digital mammography (FFDM), but it also acquires a high-energy image to visualize iodine uptake.

A retrospective study (27) comparing the diagnostic performances CESM and MRI showed CESM had similar sensitivity to MR imaging (94\% vs. 99\%), a significantly higher PPV (93\% vs. 60\%), and fewer false-positive findings ( 5 cases $v s .45$ cases). In addition, contrast-enhanced spectral mammography depicted more secondary cancers than MRI (100\% vs. 91\%).

\section{BSGI}

BSGI is also known as scintimammography or molecular breast imaging (MBI).

This modality involves injection of the radiopharmaceutical $99 \mathrm{~m}$ Tc sestamibi or $99 \mathrm{~m}$ Tc tetrofosmin 
followed by imaging with a high resolution, small fieldof-view gamma camera. At a cellular level, the radiotracer binds to mitochondria. Mitochondrial density is used as a marker of cellular proliferation and malignant cells which are rapidly proliferating would therefore take up more radiotracer than normal cells. Neovascularity from increased angiogenesis in breast cancer also leads to increased delivery of the radiotracer to the tumour. This has the advantage of detecting cancer even in mammographically dense breasts.

A systemic review and meta-analysis (28) has shown that BSGI has a high sensitivity (95\%) and specificity (80\%) in detecting breast cancer and high sensitivity (for detecting DCIS (88\%). Compared to MRI, BSGI may have lower (29), equal (30) or higher (31) sensitivity in detecting DCIS.

The impact of BSGI on surgical management has also been reported to be comparable to MRI and superior to conventional imaging with mammography and ultrasound (32). In this study, $11.9 \%$ of patients who had undergone BSGI who were initially considered to be eligible for BCS had mastectomy instead with pathology review showing these cases to be unsuitable for breast conservation. About $15.4 \%$ of patients who underwent BCS based on BSGI findings required a single re-excision due to positive surgical margins in this study.

\section{PEM}

PEM is similar to PET-CT but has higher resolution. It can be performed on patients unable to have an MRI scan. PEM uses a pair of dedicated gamma radiation detectors placed above and below the breast and mild breast compression to detect coincident gamma rays after administration of fluorine-18 fluorodeoxyglucose (18F-FDG).

When PEM has been directly compared with PET and MRI, sensitivity for detecting index lesions was comparable to MRI and superior to PET (33-36).

In ipsilateral disease, PEM showed higher specificity than MRI (34).

In contralateral disease, PEM could detect more lesions than MRI but the majority of these were assessed to be benign (36).

PEM's role in clinical practice mirrors that of MRI and in patients where MRI may be contraindicated, PEM can play a valuable role in the detection of additional foci of malignancy.

For PEM, no studies evaluating the clinical outcomes following BCS are available to date.

\section{СвСт}

This is a new technology involving a dedicated CT scanner for imaging the breast with or without contrast. The patient is prone within the scanner and one breast is scanned at a time. Most of the literature describes feasibility of the modality with little if any data on performance and outcomes compared to conventional imaging when used in a pre-operative setting.

\section{Summary}

Imaging evaluation supplementary to conventional modalities of mammography and ultrasound have not yet shown convincing improvement in clinical outcomes following BCS.

DBT and MRI may demonstrate additional lesions in the breast with MRI also showing additional contralateral disease as well. Some studies show improvement in reoperation rates compared to the control group without reduction in recurrence rates or improved disease-free survival. As a result, these cannot be recommended for all cases prior to BCS with ILC as a notable exception.

BSGI is useful as an adjunct modality to conventional imaging in detecting invasive and in-situ disease with similar sensitivity to MRI but there are few studies specifically evaluating its value as a pre-operative tool in BCS.

Further studies (such as ACRIN 6694) may demonstrate effectiveness of preoperative MRI in select patient populations, such as those with genetic mutations, and for patients with cancers with specific molecular profiles such as triple negative and Her2 positive cancers.

Utility of these additional investigations therefore depends on the individual practitioner. If there is availability of radiological expertise at the centre and if the surgeon deems that supplemental imaging modalities may be able to better aid pre-surgical planning by detecting multifocal/ multicentric disease, these may be performed in selected cases.

Newer investigations like CBCT are less widely used so far with little data evaluating their efficacy in this setting. PEM and CESM have potential with some studies showing sensitivity comparable to MRI with improved specificity and further clinical outcome studies would be awaited.

\section{Acknowledgements}

None. 


\section{Footnote}

Conflicts of Interest: The author has no conflicts of interest to declare.

\section{References}

1. Bartelink H, Horiot JC, Poortmans P, et al. Recurrence rates after treatment of breast cancer with standard radiotherapy with or without additional radiation. $\mathrm{N}$ Engl J Med 2001;345:1378-87

2. Clarke M, Collins R, Darby S, et al. Effects of radiotherapy and of differences in the extent of surgery for early breast cancer on local recurrence and 15-year survival: an overview of the randomised trials. Lancet 2005;366:2087-106

3. Veronesi U, Cascinelli N, Mariani L, et al. Twentyyear follow-up of a randomized study comparing breastconserving surgery with radical mastectomy for early breast cancer. N Engl J Med 2002;347:1227-32

4. Tot T. Subgross morphology, the sick lobe hypothesis, and the success of breast conservation. Int $\mathrm{J}$ Breast Cancer 2011;2011:634021.

5. Teboul M. Advantages of Ductal Echography (DE) over conventional breast investigation in the diagnosis of breast malignancies. Med Ultrason 2010;12:32-42.

6. Amy D, Durante E, Tot T. The lobar approach to breast ultrasound imaging and surgery. J Med Ultrason (2001) 2015;42:331-9.

7. Luparia A, Mariscotti G, Durando M, et al. Accuracy of tumour size assessment in the preoperative staging of breast cancer: comparison of digital mammography, tomosynthesis, ultrasound and MRI. Radiol Med 2013;118:1119-36.

8. Batohi B, Vinci V, Peacock C. et al. Comparison of MRI and digital breast tomosynthesis in the preoperative evaluation of multifocal breast cancer. Breast Cancer Res 2015;17: P11.

9. Krammer J, Stepniewski K, Kaiser CG, et al. Value of Additional Digital Breast Tomosynthesis for Preoperative Staging of Breast Cancer in Dense Breasts. Anticancer Res 2017;37:5255-61.

10. Houssami N, Ciatto S, Macaskill P, et al. Accuracy and surgical impact of magnetic resonance imaging in breast cancer staging: systematic review and meta-analysis in detection of multifocal and multicentric cancer. J Clin Oncol 2008;26:3248-58.

11. Brennan ME, Houssami N, Lord S, et al. Magnetic resonance imaging screening of the contralateral breast in women with newly diagnosed breast cancer: systematic review and meta-analysis of incremental cancer detection and impact on surgical management. J Clin Oncol 2009;27:5640-9.

12. Bae MS, Lee SH, Chu AJ, et al. Preoperative MR Imaging in Women with Breast Cancer Detected at Screening US. Radiology 2017;282:681-9.

13. Morris EA, Schwartz LH, Drotman MB, et al. Evaluation of pectoralis major muscle in patients with posterior breast tumors on breast MR images: early experience. Radiology 2000;214:67-72.

14. Turnbull L, Brown S, Harvey I, et al. Comparative effectiveness of MRI in breast cancer (COMICE) trial: a randomised controlled trial. Lancet 2010;375:563-71.

15. Peters NH, van Esser S, van den Bosch MA, et al. Preoperative MRI and surgical management in patients with nonpalpable breast cancer: the MONET randomised controlled trial. Eur J Cancer 2011;47:879-86.

16. Houssami N, Turner R, Morrow M. Breast Cancer: Meta-Analysis of Surgical Outcomes. Ann Surg 2013;257:249-55.

17. Sung JS, Li J, Da Costa G, et al. Preoperative breast MRI for early-stage breast cancer: effect on surgical and longterm outcomes. AJR Am J Roentgenol 2014;202:1376-82.

18. Gonzalez V, Sandelin K, Karlsson A, et al. Preoperative MRI of the breast (POMB) influences primary treatment in breast cancer: a prospective, randomized, multicenter study. World J Surg 2014;38:1685-93.

19. Fortune-Greeley AK, Wheeler SB, Meyer AM, et al. Preoperative breast MRI and surgical outcomes in elderly women with invasive ductal and lobular carcinoma: a population-based study. Breast Cancer Res Treat 2014;143:203-12.

20. Causer PA. Preoperative breast magnetic resonance imaging: controversies arising in the quest to evaluate clinical benefit. Can Assoc Radiol J 2011;62:8-14.

21. Morris EA. Should we dispense with preoperative breast MRI? Lancet 2010;375:528-30.

22. ACR practice parameter for the performance of contrastenhanced magnetic resonance imaging (MRI) of the breast. Available online: https://www.acr.org/-/media/ACR/Files/ Practice-Parameters/mr-contrast-breast.pdf

23. Marinovich ML, Houssami N, Macaskill P, et al. Metaanalysis of magnetic resonance imaging in detecting residual breast cancer after neoadjuvant therapy. J Natl Cancer Inst 2013;105:321-33.

24. Bleicher RJ, Ciocca RM, Egleston BL, et al. Association 
of routine pretreatment magnetic resonance imaging with time to surgery, mastectomy rate, and margin status. J Am Coll Surg 2009;209:180-7; quiz 294-5. Erratum in: J Am Coll Surg 2009;209:679.

25. Zhang M, Sun S, Mesurolle B. The Impact of PreOperative Breast MRI on Surgical Waiting Time. PLoS One 2017;12:e0169756.

26. Vreeland TJ, Berry Iv JS, Schneble E, et al. Routine PreTreatment MRI for Breast Cancer in a Single-Payer Medical Center: Effects on Surgical Choices, Timing and Outcomes. J Cancer 2017;8:2442-8.

27. Lee-Felker SA, Tekchandani L, Thomas M, et al. Newly Diagnosed Breast Cancer: Comparison of Contrastenhanced Spectral Mammography and Breast MR Imaging in the Evaluation of Extent of Disease. Radiology 2017;285:389-400.

28. Sun Y, Wei W, Yang HW, et al. Clinical usefulness of breast-specific gamma imaging as an adjunct modality to mammography for diagnosis of breast cancer: a systemic review and meta-analysis. Eur J Nucl Med Mol Imaging 2013;40:450-63.

29. Kim JS, Lee SM, Cha ES. The diagnostic sensitivity of dynamic contrast-enhanced magnetic resonance imaging and breast-specific gamma imaging in women with calcified and non-calcified DCIS. Acta Radiol 2014;55:668-75.

Cite this article as: Ong E. Preoperative imaging for breast conservation surgery-do we need more than conventional imaging for local disease assessment? Gland Surg 2018;7(6):554-559. doi: 10.21037/gs.2018.08.05
30. Keto JL, Kirstein L, Sanchez DP, et al. MRI versus breastspecific gamma imaging (BSGI) in newly diagnosed ductal cell carcinoma-in-situ: a prospective head-to-head trial. Ann Surg Oncol 2012;19:249-52.

31. Brem RF, Fishman M, Rapelyea JA. Detection of ductal carcinoma in situ with mammography, breast specific gamma imaging, and magnetic resonance imaging: a comparative study. Acad Radiol 2007;14:945-50.

32. Edwards C, Williams S, McSwain AP, et al. Breast-specific gamma imaging influences surgical management in patients with breast cancer. Breast J 2013;19:512-9.

33. Eo JS, Chun IK, Paeng JC, et al. Imaging sensitivity of dedicated positron emission mammography in relation to tumor size. Breast 2012;21:66-71.

34. Schilling K, Narayanan D, Kalinyak JE, et al. Positron emission mammography in breast cancer presurgical planning: comparisons with magnetic resonance imaging. Eur J Nucl Med Mol Imaging 2011;38:23-36.

35. Berg WA, Madsen KS, Schilling K, et al. Breast cancer: comparative effectiveness of positron emission mammography and MR imaging in presurgical planning for the ipsilateral breast. Radiology 2011;258:59-72.

36. Berg WA, Madsen KS, Schilling K, et al. Comparative effectiveness of positron emission mammography and MRI in the contralateral breast of women with newly diagnosed breast cancer. AJR Am J Roentgenol 2012;198:219-32. 